\title{
Werner Herzog et Alexander Kluge : cinéastes documentaires
}

\author{
J. Hamers - Université de Liège/Leiden University
}

Les documentaires de Werner Herzog et d'Alexander Kluge, et surtout la place qu'ils accordent au spectateur, démontrent à quel point leurs approches et conceptions du cinéma sont divergentes et, a priori, inconciliables. Pourtant, les deux auteurs se rejoignent sur un point essentiel rarement abordé dans les études qui leur sont consacrées. Irréductibles à une simple subjectivité exacerbée (Herzog) ou à l'idéal d'une réorganisation visuelle de la perception subjective du spectateur (Kluge), leurs démarches documentaires respectives se fondent en effet sur la perception par le spectateur d'une figure complexe qui préexiste au film tout autant qu'elle s'y déploie : le cinéaste.

The documentaries of Werner Herzog and Alexander Kluge, especially the function they are according to the spectator, demonstrates how much their approaches and conceptions of film are divergent and irreconcilable. Nevertheless, the art of these both authors have something in common that has been rarely examined by analysts who have dedicated their work to both filmmakers. More than a radical subjectivity (Herzog) or the aim of a visual reorganization of the subjective perception of the spectator (Kluge), a complex figure, and its perception by the spectator, are at the roots of their documentary practice. We call this figure the cinéaste.

\section{... que tout oppose}

Ensemble Alexander Kluge et Werner Herzog incarnent toute l'hétérogénéité définitoire du Nouveau Cinéma Allemand, tant leurs parcours respectifs, leurs films, et leurs modes de production diffèrent. Alexander Kluge est réalisateur, mais également juriste, sociologue, professeur d'université et écrivain. Ancien disciple d'Adorno, il est l'auteur de plusieurs articles et ouvrages, parfois en duo avec le sociologue Oskar Negt, qui ont marqué l'histoire de la sociologie marxiste dans les années soixante et septante en Allemagne ${ }^{1}$. On lui doit aussi une somme considérable de textes théoriques et d'essais sur le cinéma dont un grand nombre a été édité sous l'égide de l'institut de cinéma de l'école d'art d'Ulm qu'il a cofondé au début des années soixante. Enfin, il est bien évidemment l'un des principaux rédacteurs du Nouveau Cinéma Allemand, instigateur du manifeste d'Oberhausen en 1962 et concepteur du système

\footnotetext{
On songe notamment au concept «d'espace public oppositionnel» ou «espace public d'opposition», proposé par Kluge et Negt, qui fut essentiel pour toute une génération d'activistes vidéastes en Allemagne durant les années septante. Pour une traduction française d'un large extrait d'un des textes les plus importants de cette production scientifique (Oskar Negt, Alexander Kluge, Öffentlichkeit und Erfahrung, Berlin, Suhrkamp, 1972) : Oskar Negt, L'espace public oppositionnel, (trad. de l'all. par Alexander Neumann), coll. «Critique de la politique », Paris, Payot \& Rivages, 2007, pp. 55-141.
} 
de financement public que les réalisateurs de la première génération du Nouveau Cinéma Allemand vont étrenner au cours de la seconde moitié des années soixante ${ }^{2}$. Werner Herzog en revanche se définit d'abord par son autodidactisme qui va de pair avec un individualisme farouche. On ne lui connaît pas de diplômes particuliers. Il écrit peu sur son art et s'est construit au fil de ses films l'image d'un réalisateur-aventurier qui n'hésite pas à se mettre en danger pour trouver l'image absolue. Herzog pense le cinéma comme une forme d'expression quasi romantique, mystique aussi, et cherche dans ce monde l'inédit pour atteindre enfin l'extase.

Politiquement, les deux réalisateurs incarnent également des positions diamétralement opposées. On le sait, la carrière de Kluge est faite d'innombrables prises de position publiques. Sans cesse l'homme revendique, critique, conteste, et marque surtout les années soixante et septante de son empreinte alors que l'Allemagne semble encore être hantée par quelque démon totalitaire. L'engagement politique de Herzog par contre se fait beaucoup plus discret. Il est loin d'imaginer son art comme un vecteur d'émancipation culturelle, politique et intellectuelle. Et ses rares prises de position apparaissent plutôt comme des effets latents d'un non positionnement intenable selon les époques. En 1968 par exemple, il rejoint les rangs de ceux qui préfèrent retirer leurs films des festivals de Cannes ou d'Oberhausen, pour éviter seulement de servir d'emblème à un camp auquel le jeune réalisateur de l'époque ne veut en aucun cas se voir assimilé ${ }^{3}$. Et aujourd'hui encore, si nombre de ses films documentaires s'efforcent de décrire les conséquences aberrantes de l'irresponsabilité humaine, jamais Herzog ne s'associe aux documentaristes qui font des scénarii catastrophistes leur premier fond de commerce. L'heure est à l'alarme écologiste, tandis que Herzog reste ce qu'il a toujours été : un poète intrigué, perdu, fasciné, mais non révolté.

2 Cette multiplicité exceptionnelle ne va pas sans contradictions comme le constate Thomas Elsaesser qui se demande s'il existe «un véritable Alexander Kluge, ou seulement une éblouissante et, pour certains observateurs, irritante succession de déguisements, de masques et de numéros?» Thomas Elsaesser, "Mélancolie et mimétismes : les énigmes d’Alexander Kluge », (trad. de l'angl. par Pierre Rusch), Trafic, n³1, automne 1999, p. 73.

3 L'ambiguïté de ce positionnement apparaît très clairement dans son texte « Mit den Wölfen heulen » paru dans Filmkritik en juillet 1968, et dans lequel le réalisateur explique que dorénavant, il imitera ceux qui protestent en retirant leurs films d'une programmation, parce que dans un climat extrêmement polarisé, " ceux qui ne hurlent pas avec les loups, sont assimilés au camp des agneaux. » En somme, son engagement n'est ici qu'une réaction nécessaire à un contexte qui rend toute neutralité caduque. Werner Herzog, « Mit den Wölfen heulen », Filmkritik, juillet 1968, pp. 460-461. (nous traduisons) 
Enfin, il est évident que les pratiques cinématographiques des deux auteurs s'opposent également en tous points. Certes, les deux réalisateurs sont tous deux de véritables adeptes de la documentarisation du cinéma de fiction. Mais leurs façons de penser une interpénétration des genres paraissent inconciliables. On se souvient des campagnes de promotion et d'autopromotion de Aguirre, la colère de Dieu ${ }^{4}$ (W. Herzog, 1972) ou encore de Fitzcarraldo $^{5}$ (W. Herzog, 1982), qui faisaient l'apologie de tournages extrêmes marqués par une interférence périlleuse entre réalité du plateau et univers diégétique. Aux yeux de bon nombre de critiques mais aussi du réalisateur lui-même, cette interférence dotait les films d'une véritable plus-value d'authenticité. Fitzcarraldo par exemple valait autant pour la qualité de sa mise en scène et de son scénario que pour l'engagement de son acteur principal, Klaus Kinski, qui avait refusé toute doublure, même dans les situations les plus dangereuses ${ }^{6}$. Pour Kluge en revanche, la rencontre de situations prélevées dans la vie réelle et d'actions mises en scène dans ses films de fiction, essentielle tout au long de sa première période songeons seulement à Anita $G^{7}$ (A. Kluge, 1966) ou à Travail occasionnel d'une esclave $^{8}$ (A. Kluge, 1973) - est proprement idéologique voire politique :

« Ici aussi il s'agit du mélange [litt. « forme de mélange », ndtr.] qui, entre document et fiction, entre montage et reproduction intégrale, entre fantaisie et sens de la réalité, est vecteur de transmission. Contrairement à ce que l'on admet, la sociologie et les contes ne sont pas des éléments contradictoires. Ce sont deux pôles appartenant à une seule et même chose qui change d'apparence, suivant que l'on tient compte de la capacité de l'être humain à tenir le coup face aux faits, ou de sa capacité à élaborer des souhaits. [...] Cela [le travail à l'institut d'Ulm, ndtr.] implique une certaine conception du réalisme, notamment d'un réalisme qui prend autant la fantaisie et les souhaits des êtres humains au

4 DVD : Aguirre, la colère de Dieu, in Werner Herzog (coffret 5 DVD), vo. s-t. fr., s.1., Werner Herzog Filmproduktion, s.d.

5 DVD : Fitzcarraldo, in Fitzcarraldo, La ballade de Bruno (coffret 2 DVD), vo. s-t. fr., Paris, coll. « Les films de ma vie », 2006.

6 Le documentaire Ennemis intimes (W. Herzog, 1998 ; DVD : Ennemis intimes, in Herzog - Kinski. Cobra Verde \& Ennemis intimes (coffret 2 DVD), vo. s-t. fr., Potemkine Editions, 2009), consacré à la collaboration entre Herzog et Kinski, est quasi exclusivement voué à l'apologie de cette forme d'authenticité que le réalisateur croit trouver dans la rencontre entre réalité du tournage et diégèse. Emmanuel Carrère comprend " la conception du tournage » de Herzog « comme immersion totale de l'esprit, mais aussi du corps, dans la matière même qu'il s'agit de façonner, comme exploit physique où le génie s'acquiert au prix de l'endurance et de la volonté [...]. » Emmanuel Carrère, Werner Herzog, coll. « Cinégraphies », Paris, Edilig, 1982, p. 70. Au sujet du tournage de Fitzcarraldo et du refus de tout trucage, voir aussi Edgar Reitz, «Werner Herzog », in Edgar Reitz, Bilder in Bewegung. Essays. Gespräche zum Kino, Reinbek, Rowohlt, 1995, p. 70.

7 DVD: Abschied von Gestern \& Gelegenheitsarbeit einer Sklavin, vo. s-t. fr., Munich, Edition Filmmuseum, 2007.

8 Idem 
sérieux, que le monde des faits. Les faits seuls ne sont pas réels, les souhaits en eux-mêmes non plus ${ }^{9}$.»

En somme, pour Herzog, les incursions d'une certaine «documentarité » dans la fiction doivent attester l'engagement réel d'une équipe de tournage entièrement dévouée aux (mégalo)manies d'un auteur visionnaire. Tandis que pour Kluge, l'interpénétration des deux genres vise une plus grande « authenticité » et effectivité politique de ses films.

\section{Deux subjectivités}

Si on s'intéresse plus spécifiquement à leurs films documentaires, l'opposition entre Herzog et Kluge devient patente. Pour le premier, le cinéma documentaire n'est authentique que s'il renonce ou résiste à la tentation d'une objectivité illusoire que semble lui offrir le cinéma direct ou le cinéma vérité. Dans un de ses rares textes aux allures d'essais, La déclaration du Minnesota ${ }^{10}$, Herzog affirme en des termes parfois obscurs, ce refus d'une fausse objectivité :

1. A force de se déclarer comme tel, ce qu'on appelle Cinéma Vérité est dénué de vérité. Il n'accède qu'à une vérité superficielle, à la vérité des comptables.

2. Un représentant connu du Cinéma Vérité déclara publiquement qu'il était facile de trouver la vérité en prenant une caméra et en essayant d'être honnête. Il ressemble à ce gardien de la Cour Suprême qui s'indigne du nombre de lois écrites et des procédures légales. "Pour moi », dit-il, «il ne devrait y avoir qu'une seule loi : les mauvais gars devraient aller en prison ». Malheureusement, il a en partie raison, la plupart du temps, dans la plupart des cas.

3. Le Cinéma Vérité confond faits et vérité, et ainsi, ne laboure que des pierres. Malgré tout, les faits ont parfois un pouvoir étrange et bizarre qui fait que leur vérité inhérente parait incroyable.

4. Le fait crée des normes, la vérité, l'illumination.

9 Alexander Kluge, "Ein Hauptansatz des Ulmer Instituts », in Klaus Eder, Alexander Kluge, Ulmer Dramaturgien. Reibungsverluste. Stichwort: Bestandsaufnahme, (Mit Beiträgen von Günther Hörmann, Reinhard Kahn, Michel Leiner, Maximiliane Mainka, Wielfried Reinke, Edgar Reitz, Peter Schubert), Munich, Vienne, Carl Hanser Verlag, 1980, p. 7. (nous traduisons) Voir aussi: Alexander Kluge, "Reibungsverluste », in Klaus Eder, Alexander Kluge, idem., p. 93; Jan Dawson, Alexander Kluge, « An Interview With Alexander Kluge. But Why Are the Questions so Abstract?», in Jan Dawson, Alexander Kluge \& The Occasional Work of a Female Slave, New York, Zoetrope, 1977, p. 35 (Première édition : «Alexander Kluge », Film Comment, vol. 10, n6, 1974.).

10 Werner Herzog, «Minnesota Declaration. Truth and Fact in Documentary Cinema », 1999. Le lecteur trouvera une reproduction intégrale du texte original en anglais sur le site officiel de Werner Herzog (dernière consultation le 15 avril 2011). URL : http://www.wernerherzog.com/52.html\#c93 
5. Il y a une couche plus profonde de vérité au cinéma, et il existe quelque chose comme une vérité poétique, extatique. Cela est mystérieux et insaisissable, et ne peut être atteint que par la fabrication, l'imagination et la stylisation ${ }^{11}$.

Pour le réalisateur munichois, le seul moyen de saisir la vérité consiste à assumer, et à révéler, la totale subjectivité du réalisateur. Sa démarche documentaire se fonde donc en quelque sorte sur un doute lucide originel proprement cartésien : si rien ne permet d'atteindre le réel étant donné que le réalisateur est nécessairement toujours déjà rivé à sa propre subjectivité, la seule voie pour tendre vers la vérité réside dans une représentation documentaire qui résulte directement de cette subjectivité, c'est-à-dire d'une perception tout à fait personnelle et subjective du réel. Chez Herzog, cette conception radicale de la pratique documentaire peut aller jusqu'au mensonge, conséquence assumée d'une perception fondamentalement égocentrée du monde.

Le documentaire Leçons des ténèbres ${ }^{12}$ (W. Herzog, 1992), qui a également donné son titre à la déclaration citée précédemment ${ }^{13}$, exemplifie de façon quasi didactique cet intégrisme du subjectif. Dans ce documentaire tourné au Koweït après la première Guerre du Golfe, l'univers visuel et acoustique s'apparente à un monde dans lequel toute communication est devenue impossible, un monde dans lequel les repères spatio-temporels ont été abolis, un monde enfin qui oscille en permanence entre genèse et apocalypse. Accompagnant de longues vues aériennes qui, au son de Mahler ou Wagner, semblent attester l'existence d'un point de vue planant sur le désert koweïtien, le réalisateur commente lui-même les images et «ment » à plusieurs reprises à un spectateur qui n'a d'autre choix que d'adhérer aux lubies de l'auteur ${ }^{14}$. Dans l'optique de Herzog, ces entorses à la réalité objectivable sont pleinement justifiées ${ }^{15}$. Car dans un monde où il est devenu difficile de communiquer, de distinguer entre

11 Nous reprenons ici la traduction proposée par Valérie Carré. Valérie Carré, La quête anthropologique de Werner Herzog. Documentaires et fictions en regard, coll. «Faustus/Etudes germaniques », Strasbourg, Presses universitaires de Strasbourg, 2007, pp. 209-210.

12 DVD : Lessons of Darkness, in Documentaries 1962-2005 (coffret 13 DVD), vo. s-t. fr., Vienne, Werner Herzog Filmedition, 2010.

13 Leçons des ténèbres est le sous-titre de la déclaration du Minnesota.

14 Ainsi, dans le chapitre 12, alors que des pompiers enflamment des geysers de pétrole qu'ils viennent d'éteindre le réalisateur nous dit: "Maintenant ils sont de nouveau satisfaits. Maintenant ils ont à nouveau quelque chose à éteindre. " Auparavant, le commentaire a mis ce geste sur le compte de la folie des pompiers qui rallument cela même qu'ils ont péniblement éteint : "Pris de folie, d'autres les imitent. » Il va de soi que l'interprétation donnée par le réalisateur au geste des pompiers résiste à la raison. En réalité, ces pompiers rallument les puits éteints afin de prévenir l'apparition de poches de gaz et de lacs artificiels durant la nuit.

15 Dans un entretien portant sur un autre documentaire de Werner Herzog, Le pays du silence et de l'obscurité (W. Herzog, 1971), le réalisateur déclare : « [...] cela ne me dérange pas d'aller jusqu'au bord du mensonge, 
le haut et le bas, entre l'eau et le pétrole, entre le ciel, la terre et ses reflets, il serait absurde de vouloir encore mesurer la correction d'une information à la seule aune de sa correspondance avec un hypothétique réel (re)connu ${ }^{16}$. Et il ne reste donc au réalisateur qu'à rendre le réel tel qu'il le perçoit, le comprend, se le raconte au moment où il le regarde. Le spectateur est, quant à lui, totalement subordonné à cette seule vision du monde, à cette seule vision. Il en est réduit à adopter une posture passive. Il n'a d'autre choix que de re-voir comme l'auteur a vu.

Pour Alexander Kluge en revanche, le spectateur est une entité qui participe pleinement et activement à la construction d'un documentaire. Sa subjectivité mais surtout ses souvenirs visuels déterminent le sens produit par le film dans la même proportion que le regard et les intentions du réalisateur. Pour Kluge, l'auteur doit assembler un ensemble de représentations qui ne rendent que partiellement le réel dont il est question dans le film. Ces images renvoient ensuite le spectateur vers d'autres images, non montrées. Ce renvoi déclenche alors un ensemble d'associations révélatrices qui transforment le public en une seconde instance auctoriale. Par un retour sur la mémoire du spectateur, le film peut donc convoquer un ensemble de représentations et de problématiques qui dépassent de loin les seules images du film ${ }^{17}$. Pour Edgar Reitz, ce cinéma dit «des associations » projette le spectateur dans un tourbillon vertigineux de significations :

Le spectateur ne se fait pas interpeller, de façon neutre, séduit par l'illusion. Le spectateur ne se fait pas conduire dans un espace clos. Il n'est pas projeté dans un

pour dévoiler une forme de vérité plus intense. » Hans-Günther Pflaum, « Bilder vom Zivilisationsstand: Werner Herzog», in Jörg-Dieter Kogel, Europäische Filmkunst. Regisseure im Porträt, coll. "Fischer Cinema», Francfort, Fischer Taschenbuch Verlag, 1990, p. 85. (nous traduisons) Sur le mensonge et l'opposition au cinéma vérité, voir aussi : David Davidson, « Borne Out of Darkness : The Documentaries of Werner Herzog », Filmcriticism, vol. 5, n 1, automne 1980, pp. 10-25.

16 Pour une analyse plus fouillée des liens forts entre les décors du film et le mensonge documentaire, on lira notamment: Alexander Schwarz, «Wahre Bilder des Grauens. Ästhetik und Authentizität in Werner Herzogs 'Lektionen in Finsternis' (1992) », in Manfred Hattendorf (éd.), Perspektiven des Dokumentarfilms, Munich, Diskurs Film Verlag Schaudig \& Ledig, 1995, pp. 167-190; Jeremy Hamers, "Lektionen in Finsternis de Werner Herzog. Quelle esthétique documentaire après la guerre ? », in François-Emmanuel Boucher, Sylvain David, Janusz Przychodzen, La Paix. Esthétiques d'une éthique, Bern, Peter Lang, 2007, pp. 19-35.

17 « Au sens klugien du terme, le film d'auteur est un ensemble de formes d'expression qui visent, par le biais des possibilités du cinéma, à organiser la réception du spectateur-auteur, le réalisateur égarant simultanément son public par des formes d'un «montage sauvage» [en français dans le texte] qui veut susciter et faire émerger fantaisie et associations chez le récepteur. »Werner Barg, « Ein Dokumentarist des Protestes. Alexander Kluges Theorie des Dokumentarfilms. Beobachtungen $\mathrm{zu}$ seinen Essay-Filmen und Fernsehmagazinen », in Manfred Hattendorf (éd.), op. cit., p. 113. (nous traduisons) 
système de rapports immuables. Le spectateur, libéré, est par contre confronté à une un ensemble inouï de significations multiples ${ }^{18}$.

D’un côté, Werner Herzog, réalisateur a priori apolitique, mégalomane, menteur, adepte d'une subjectivité exacerbée en vertu de laquelle le spectateur est cantonné dans l'observation passive des perceptions oniriques et des déformations du réel imaginées par l'auteur. De l'autre, Alexander Kluge, cinéaste rationnel, marxiste, obsédé par les enjeux historiques et politiques des images qu'il assemble. Contrairement à Herzog, il accorde une place prépondérante à la subjectivité du spectateur. La mémoire d'images plus anciennes doit en effet guider ce dernier vers une compréhension supérieure des enjeux politiques et historiques que le film seul ou la subjectivité du seul auteur seraient incapables de faire émerger. Dépassant alors l'épiderme de la représentation réaliste, le spectateur peut s'ouvrir à une véritable perception critique du monde. Posées de la sorte, les conceptions du documentaire de Herzog et de Kluge paraissent totalement incompatibles. Pourtant, leurs ouvres documentaires et surtout la réception spectatorielle dont elles sont pour partie les produits, entretiennent certaines similitudes étonnantes.

\section{Mensonge et vérité}

Je pourrais dévorer Fata Morgana de Herzog. Tel un cannibale je pourrais le dévorer toujours et à nouveau. [...]

Edgar Reitz : Que trouves-tu dans le désert?

Alexander Kluge : Un pays vide. On dit: Tu ne représenteras pas Dieu. Cela est présent dans le désert, dans l'Antarctique, dans le ciel, dans le lointain ${ }^{19}$.

A rebours de l'opposition établie jusqu'ici, cette déclaration d'Alexander Kluge au sujet de Fata Morgana $^{20}$ (W. Herzog, 1969) semble augurer une réelle proximité entre les deux

18 Edgar Reitz, « Utopie Kino », in Edgar Reitz, Die Liebe zum Kino. Utopien und Gedanken zum Autorenfilm, Cologne, Verlag Köln 1978, 1983, p. 20. (nous traduisons) Selon Reitz, ce cinéma, qu'il qualifie avec Kluge de «cinéma analytique » est affranchi de la catégorisation habituelle des formes et discours pour laisser la place à un type cinématographique nouveau : «Celui-ci est totalement externe aux catégories habituelles. Il se sert de toutes les possibilités techniques, stylistiques et méthodologiques des genres cinématographiques connus jusqu'alors, pour atteindre un but qui peut être décrit grossièrement par le terme 'analyse'. » Idem, p. 18. Le lecteur trouvera quelques réalisations quasi didactiques et explicites de ces associations dans les séquences documentaires coréalisées par Volker Schlöndorff qui ouvrent et qui referment le film collectif L'Allemagne en automne (R.W. Fassbinder, V. Schlöndorff, A. Kluge, E. Reitz, 1978).

19 Edgar Reitz, « Alexander Kluge », in Edgar Reitz, Bilder in Bewegung, op. cit., p. 84.

20 DVD : Fata Morgana, in Documentaries 1962-2005 (coffret 13 DVD), vo. S-t. fr., Vienne, Werner Herzog Filmedition, 2010. 
réalisateurs. L'emploi du terme «cannibale» d'abord nous renvoie à une intrication essentielle entre les cinémas de ceux que nous avons opposés jusqu'à présent. Kluge adhère littéralement au cinéma de Herzog, au point de se retrouver dans son film, de le considérer comme un semblable, un semblable que l'on dévore ${ }^{21}$. L'énumération de quelques lieux de tournage (le désert, l'antarctique, le ciel) trahit quant à elle l'existence d'obsessions communes aux deux auteurs. Ces décors sont en effet, faut-il le rappeler, ceux-là mêmes que Herzog affectionne tout particulièrement pour le tournage de ses documentaires ${ }^{22}$.

La proximité entre les deux auteurs qu'annonce ce bref extrait d'interview se trouve confirmée par d'autres déclarations de Kluge, qui font l'apologie du mensonge comme voie vers la vérité. Dans ces passages, Kluge semble véritablement paraphraser la position de Herzog sur le sujet :

Edgar Reitz: Donc je ne peux pas mesurer l'image de cinéma à la réalité.

Alexander Kluge: Non. La vérité est en quelque sorte une notion théologique. [...] Quand tu commences à mentir, tu es lié et asservi à la réalité, à la vérité si tu veux. La plupart du temps, un menteur est bien plus en mesure de traiter avec la vérité que celui qui recherche la vérité. Comment pourrait-il constater ce qui est vrai ? Le menteur, lui, le sait très précisément. Il vérifie en permanence si son mensonge résiste à ce qui peut être tenu pour vrai ${ }^{23}$.

Cette façon de penser le cinéma et son rapport au réel, induit nécessairement un glissement du contrat tacite - qui subsiste aux yeux de certains - entre le spectateur et le réalisateur de documentaires. Car si la conscience du documentariste l'amène à mentir et à chercher au lieu de trouver la vérité, à préférer le mensonge plus proche de la vérité à tout autre discours, et si le spectateur est en mesure de le savoir, la position de ce dernier s'en verra nécessairement bouleversée.

Cette hypothèse et son affûtage risquerait bien évidemment de nous entraîner dans une oscillation irrésolue, qui conduirait à ce que nous nommerions provisoirement une paradoxale

21 Cette déclaration de Kluge au sujet de Fata Morgana (W. Herzog, 1969) est d'autant plus remarquable que le film concerné peut être considéré comme un véritable archétype du documentaire herzogien, avec ses paysages post-apocalyptiques, ses personnages dont la réalité est plus que douteuse, et de longs plans séquences tournés par un documentariste visionnaire fait prisonnier de paysages lunaires infinis.

22 Le désert est en effet au centre de plusieurs de ses films documentaires (Fata Morgana, Leçons des ténèbres, etc.). Le ciel en tant qu'espace obsède littéralement Herzog (The Wild Blue Yonder, 2005 ; The White Diamond, 2004), tandis que l'antarctique est la région dans laquelle il a tourné en 2007 un de ses derniers documentaires, Rencontres au bout du monde. DVD : The Wild Blue Yonder, in Documentaries 1962-2005 (coffret 13 DVD), vo. S-t. fr., Vienne, Werner Herzog Filmedition, 2010; DVD: The White Diamond, idem.

23 Edgar Reitz, « Alexander Kluge », op. cit., p. 88. (nous traduisons) 
crédulité lucide. Nous devrions alors imaginer un spectateur qui saurait, et qui, malgré tout, accepterait que ce qu'il voit là est bien au documentaire étant donné que tout dans le film oriente sa réception dans ce sens. Mais ce risque nous encourage aussi à déchirer le carcan sémio-pragmatique auquel il a été fait référence ici, pour nous intéresser encore à une toute autre relation auteur/spectateur, qui dépasse de loin le seul contrat tacite entre un énonciateur et un récepteur.

\section{Au-delà des antagonismes : le cinéaste}

Dans A Cinema Without Walls, Timothy Corrigan avance que la figure de l'auteur, certes fondée sur un ensemble de traits stylistiques, doit aussi être interrogée à la lumière de la relation établie avec le spectateur. Précédant le film, l'auteur prédétermine en effet la réception de son oeuvre :

To view a film as the product of an auteur means to read or to respond to it as an expressive organization that precedes and forecloses the historical fragmentations and subjective distortions that can take over the reception of even the most classically coded movie ${ }^{24}$.

Pour Corrigan, l'auteur serait donc une entité qui se manifeste entre autres en amont de l'œuvre et dont les discours dirigent la lecture qu'en fera le spectateur. Pour expliciter cette idée, l'analyste convoque entre autres les exemples de Herzog et de Spielberg :

To respond to a movie as primarily or merely a Spielberg film is, after all, the pleasure of refusing an evaluative relation to it - a pleasure that might be equally true of the standard reception of Herzog movies - and much of that pleasure lies in being able to already know, not read, the meaning of the film in a totalizing image that precedes the movie in the public images of its creator. An auteur film today seems to aspire more and more to a critical tautology, capable of being understood and consumed without being seen ${ }^{25}$.

\footnotetext{
24 Timothy Corrigan, A Cinema Without Walls. Movies and Culture After Vietnam, New Brunswick, Rutgers University Press, 1991, p. 102.

25 Timothy Corrigan, op. cit., p. 106.
} 
La réception du spectateur est donc déterminée en amont du film par l'image qu'il se fait de l'auteur ${ }^{26}$. Cette image est le produit d'un ensemble de métadiscours que l'analyste localise par exemple dans les making of, les interviews et les discours oraux de réalisateurs qui font escorte à une projection. Et même si Corrigan cantonne sa réflexion dans le seul champ de la fiction, on pourrait a priori reconnaître dans son auteur un « énonciateur réel » qui, pour ne pas affecter l'illusion fictionnelle, se placerait systématiquement hors du film, dans un lieu indéterminé et à partir duquel il guide la réception spectatorielle. Pourtant, au contact du cinéma documentaire, la comparaison à cet énonciateur tel qu'il a été défini jadis par les théories sémio-pragmatiques, se révèle totalement insuffisante et occulte de toute évidence une facette de l'auteur de documentaires qu'il s'agit de repenser en d'autres termes.

Dans le cas du cinéma documentaire, l'auteur corriganien n'est pas assimilable à l'énonciateur supposé réel, au caméraman, ou à un spécialiste interviewé. Car chez Corrigan, sa présence n'enfante en aucun cas une instance nécessairement énonciative et surtout elle n'est pas nécessairement avérée dans le discours filmique lui-même (ce qui permet à Corrigan d'imaginer le film comme un objet second pour la réception spectatorielle). Or, si le discours du documentariste peut, à l'instar de celui de l'auteur de Corrigan, effectivement préexister au film, il se déploie nécessairement aussi à l'intérieur de l'œuvre, précisément sous la forme d'une présence diffuse et non avérée. Et c'est d'ailleurs pour cette raison que, contrairement aux fictions envisagées par Corrigan, le documentaire ne peut en aucun cas devenir un objet second, dispensable pour son spectateur.

A la notion d'auteur, nous préférerons dès lors la notion de cinéaste qui, en documentaire, préexiste au film, mais s'y déploie aussi nécessairement sans pour autant que ces deux manifestations ne renvoient au seul personnage réel qui serait à l'origine du film. Plus qu'une autorité intellectuelle ou physique, le cinéaste se définit avant tout par un regard dans le monde et sur le monde. Ce regard n'est pas réductible à la seule réalité (physique ou morale) de l'énonciateur. Et il est tout à la fois le produit et le premier instigateur d'une relation entre le film et son spectateur. Cette relation reconfigure la réception spectatorielle qui n'est plus exclusivement déterminée par les métadiscours et discours émanant d'un sujet réel placé avant ou dans le film. Le regard du cinéaste peut en effet préexister au film dans l'esprit du

26 Cette réflexion s'inscrit chez Corrigan dans le cadre très clair d'une critique de ce qu'il nomme le « commerce de l'auteursime», démontrant entre autres que l'instance de l'auteur est devenue le premier vecteur de promotion d'un film, qui prévaut sur le discours filmique lui-même. 
spectateur. Mais il peut aussi se déployer à l'intérieur du film, et toucher dès lors un spectateur vierge de tout contact avec l'auteur ${ }^{27}$. Enfin, ce regard s'adjoint aussi la complicité d'un autre regard (et donc d'une autre subjectivité), celui du spectateur sur le film.

Les films documentaires des réalisateurs qui nous intéressent ici se rejoignent dans cette figure du cinéaste. Irréductible à une simple subjectivité exacerbée (Herzog) ou à l'idéal d'une réorganisation visuelle de la perception subjective du spectateur (Kluge), le regard du documentariste suscite la reconnaissance non pas d'un « auteur-individu », mais d'une figure plus complexe qui n'est pas nécessairement réelle ou supposée réelle. Cette figure est le produit de la subjectivité de l'auteur (regard) et de celle d'un spectateur qui se fait le relais de ce regard. Face à un documentaire de Herzog, le spectateur voit « du Herzog », c'est-à-dire le fruit d'un regard herzogien sur le monde, qui peut induire une part de mensonge consenti (c'est-à-dire aussi senti avec) par le spectateur. Certes, ce dernier n'oublie pas le réalisateur mégalomane qui prétend se mettre en danger pour trouver l'image rare. Mais il sait aussi que ce regard ne doit pas être évalué au seul prisme du respect d'un fait objectivable. En ce sens, le contrat tacite réalisateur/spectateur qui disqualifiait aux yeux de certains la démarche du réalisateur munichois, doit être redéfini. Il s'apparente à présent à une forme de complicité induite par un cinéaste qui compte sur, tout autant qu'il fonde, une réception spécifique de son film (et qui dépasse de loin la seule alternative documentaire/fiction). De la même façon, le spectateur d'un documentaire de Kluge sait que les images montrées servent en réalité à une réarticulation de représentations anciennes et contemporaines, tournées pour les besoins du film ou tirées d'archives. Ici aussi, il ne les considérera pas comme de simples représentations. Il les consommera plutôt en tant qu'éléments partiels d'un discours supérieur qui s'appuie intégralement sur un ensemble d'associations dont il est le premier producteur.

Nous l'avons dit, cette complicité entre le regard du documentariste et celui du spectateur n'est pas le seul fait d'un discours qui précéderait le film. Elle est également construite par le film lui-même. Entre autres par le biais des voix off des deux cinéastes. On le sait, Herzog et Kluge lisent bon nombre de leurs commentaires eux-mêmes ${ }^{28}$. Leurs voix sont reconnaissables entre mille. Kluge possède un timbre doux, développe une diction précise,

\footnotetext{
27 On peut donc imaginer un spectateur qui, malgré une éventuelle et fort improbable virginité, s'engagerait d'emblée dans une relation particulière avec le cinéaste, c'est-à-dire le perçoive comme un regard spécifique qui oriente sa réception de l'oeuvre.

28 Herzog a même été jusqu'à lire aussi les commentaires pour les versions anglaises de ses films.
} 
adopte un débit rapide, et une intonation quasi enfantine. Chez Herzog par contre, le timbre est grave et rocailleux, parfois presque rauque. Son débit est lent et hésitant, son intonation affectée et anxiogène. Par sa voix, Herzog le tourmenté suggère que le moment est grave, que nous voyons de l'inédit, et que cet inédit a été obtenu aux prix de sacrifices potentiels ou réels qui dotent le filmé d'une plus-value certaine. Par sa voix et ses commentaires, il a créé un véritable moment sacré. Un moment qui se trouve au-delà de tout mensonge ou de toute vérité ordinaire. Dans le cas de Kluge, la lecture apparemment ingénue de quelques commentaires encourage par contre le spectateur à adopter un regard analytique. Les paroles du cinéaste ne servent un discours explicite qu'en apparence. Et le spectateur comprend alors que ses images ne sont pas réductibles à ce qu'elles représentent.

Arrivé au terme de cette brève réflexion, les contours du cinéaste restent vagues. Les voix n'en sont que les manifestations les plus évidentes. Et nous savons seulement qu'il n'est ni pure construction du spectateur, ni figure imposée en amont du film. Mais nous pouvons déjà affirmer qu'une définition de cette figure s'appuiera nécessairement, nous espérons en avoir convaincu le lecteur, sur la rencontre de deux subjectivités : celle d'un auteur qui par ses diverses manifestations avant et dans le film instaure une relation particulière avec le spectateur; celle du spectateur affranchi de sa foi en un énonciateur réel, qui peut enfin comprendre le film comme la partie intégrante d'un regard sur le monde. Ce regard ne produit plus des représentations. Il contribue à la construction de ce qu'il conviendra désormais d'appeler des objets visuels, fruits d'une rencontre (plus que d'une juxtaposition concurrentielle) de plusieurs subjectivités. 